\title{
A serendipitous discovery: an adult case of congenital generalized lipodystrophy
}

\author{
Abdul Rehman Zia Zaidi, ${ }^{1,2}$ Asma Sikander, ${ }^{2}$ Eissa Faqeih, ${ }^{3}$ Njoud Abdulrahman Alhowar, ${ }^{1,4}$ Mohammed AlSheef ${ }^{2}$ \\ ${ }^{1}$ College of Medicine, Alfaisal University; ${ }^{2}$ Department of Medicine, King Fahad Medical City; ${ }^{3}$ Section of Medical Genetics, \\ Children's Specialist Hospital, King Fahad Medical City; ${ }^{4}$ Department of Emergency Medicine, King Fahad Medical City, \\ Riyadh, Saudi Arabia
}

\begin{abstract}
Congenital generalized lipodystrophies are a heterogeneous group of rare disorders characterized by loss of subcutaneous fat, muscular hypertrophy, acanthosis nigricans, hepatomegaly, cardiac arrhythmias, impaired metabolism, and mental retardation. We report a 41-year-old female that presented as a rare adult case of congenital generalized lipodystrophy type 1 . She was referred to us as a case of hypertriglyceridemia with features of generalized lipodystrophy and manifestations of associated multiple metabolic disorders. Triglyceride level was tremendously high with reduced high-density lipoproteins. The patient was found to have a pathogenic novel mutation in the AGPAT2 gene, confirming the diagnosis of congenital generalized lipodystrophy type 1 .
\end{abstract}

Correspondence: Abdul Rehman Zia Zaidi, Department of Medicine, King Fahad Medical City, P.O. Box 59046, Riyadh 11525, Saudi Arabia.

Tel.: +966.568104818 - Fax: +966.11.2176199.

E-mail: ar-zia@hotmail.com

Key words: Hypertriglyceridemia; Berardinelli Seip congenital lipodystrophy; lipodystrophy; phlebomegaly; AGPAT2.

Acknowledgments: the authors would like to thank and acknowledge King Fahad Medical City (KFMC) and their Research Office for tremendous support and IRB of KFMC for ethical approval of the study.

Contributions: all the authors equally contributed to the conduct of the study and preparation of the manuscript.

Conflict of interests: the authors declare no potential conflict of interests.

Ethics approval and consent to participate: the patient agreed to sign a consent form for her participation in the research presented in this manuscript with full knowledge of the possible risks and benefits of participation. The study was approved by the Institutional Review Board of King Fahad Medical City, Riyadh, Saudi Arabia (IRB Log Number: 19-164). The study was conducted in accordance with recommendations of the International Conference on Harmonization for Good Clinical Practice (ICH-GCP).

Received for publication: 6 November 2019.

Revision received: 1 December 2019.

Accepted for publication: 20 December 2019.

This work is licensed under a Creative Commons Attribution NonCommercial 4.0 License (CC BY-NC 4.0).

${ }^{\circ}$ Copyright: the Author(s), 2020

Licensee PAGEPress, Italy

Italian Journal of Medicine 2020; 14:31-35

doi:10.4081/itjm.2020.1231

\section{Introduction}

Congenital generalized lipodystrophy (CGL) or Berardinelli Seip congenital lipodystrophy (BSCL) is heterogeneous and rare autosomal recessive disorder characterized by a noticeable absence of fat, acromegalic features and severe metabolic derangements such as insulin resistance and dyslipidemia. Diabetes mellitus generally develops during the second and third decades. ${ }^{1}$ The estimated worldwide prevalence is one in 10 million. ${ }^{2}$ CGLs are classified into four types based on their molecular etiologies. CGL type 1 (CGL1) is caused by homozygous or compound heterozygous mutation in the gene encoding 1-acylglycerol-3-phosphate O-acyltransferase-2 (AGPAT2). Type 2 is caused by a mutation in the $B S C L 2$ gene, type 3 is caused by a mutation in the $C A V 1$ gene, and type 4 is caused by a mutation in the PTRF gene. Patients with a mutation in AGPAT2 harbor homozygous or compound heterozygous mutation that subsequently leads to defect in the 1-acylglycerol-3-phosphate-O-acyltransferase responsible for catalyzing the conversion of lysophosphatidic acid to phosphatidic acid. We describe a 41-year-old female with a CGL1 variant presenting with severe hypertriglyceridemia.

\section{Case Report}

Informed consent was obtained from the patient and her family for the purpose of publication. We explained to them the importance of a case report, research, and its impact on clinical practice. Institutional Review Board (IRB) approval for this publication was also taken. 
A 41-year-old female born out of first-degree consanguineous marriage was referred to our clinic for management of hypertriglyceridemia. She presented with enlarged hands and feet, generalized loss of subcutaneous fat, prominent muscle appearance, and prominent subcutaneous veins in her upper and lower limbs bilaterally (Figure 1). She reported a history of diabetes mellitus type 2 requiring insulin therapy for the last 12 years complicated by diabetic nephropathy, uncontrolled hypertension, left-sided breast cancer T4N1M0 status post-mastectomy for which she was on a course of tamoxifen and goserelin. She also reported a history of secondary amenorrhea, with her last menstrual period being at the age of 18 . She complained of having yellowish nodules on her fingers as well. This patient's family had a prominent history of consanguinity with the patient's parents being first-degree relatives, and grandparents were also first-degree relatives. One of her paternal uncles had history of the same disease. One of her brothers had a similar presentation. She has four brothers and two sisters that are healthy and asymptomatic. In this patient's pedigree, we can see that the mode of inheritance of this disease is autosomal recessive (Figure 2).

On examination, she was alert with vital signs

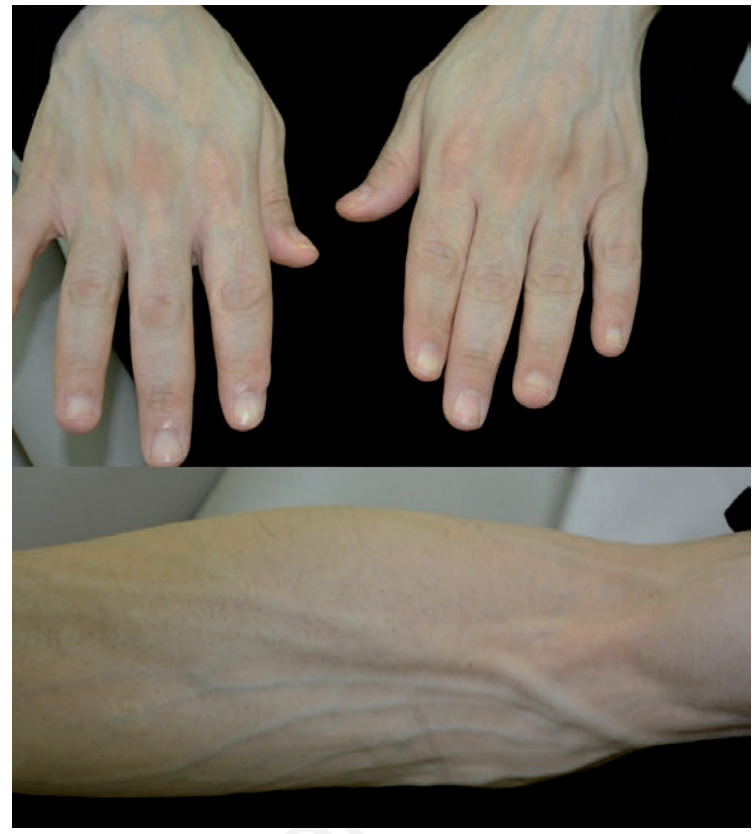

Figure 1. Prominent muscles with loss of subcutaneous fat and phlebomegaly.

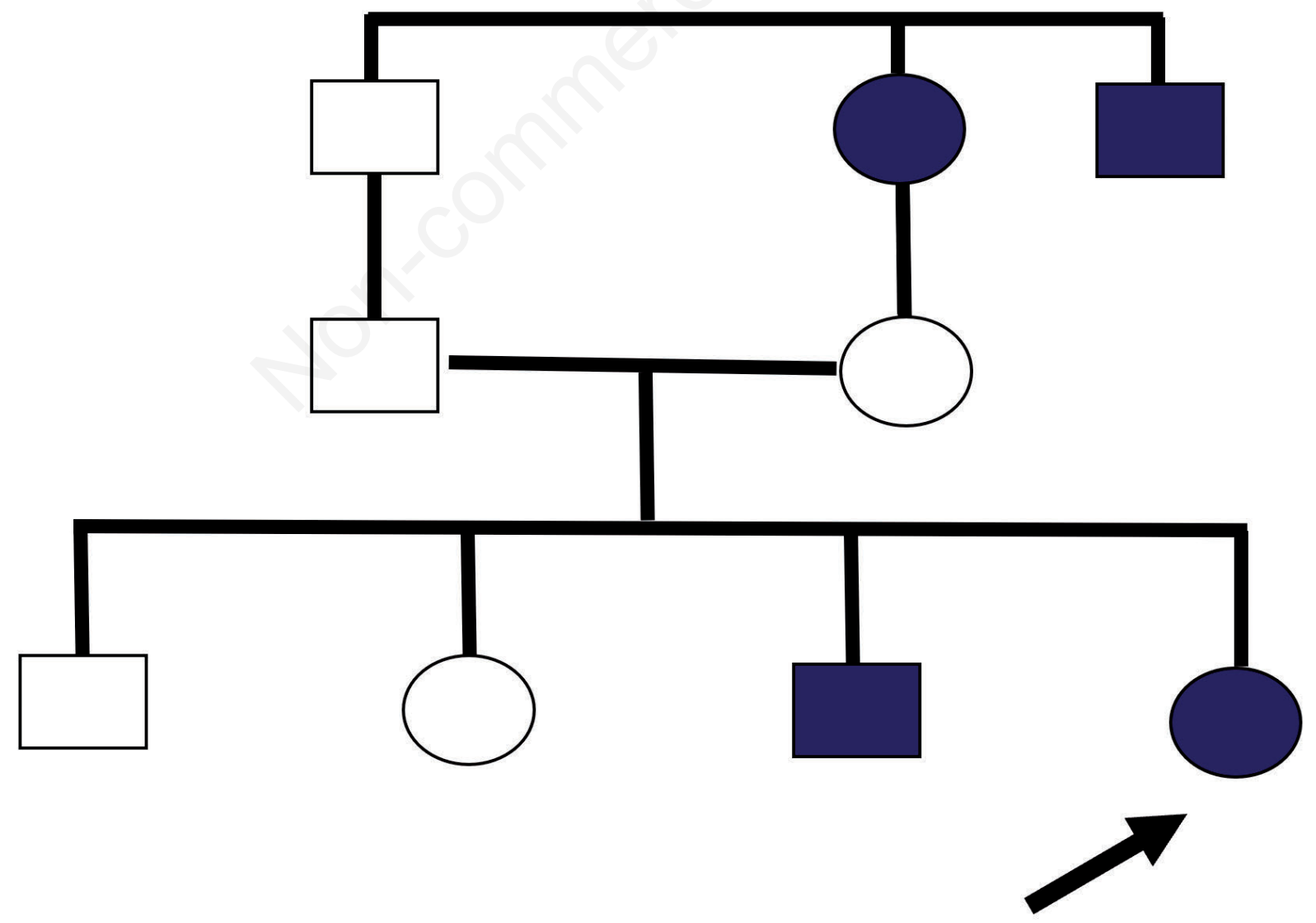

Figure 2. Patient's family pedigree composite indicating an autosomal recessive pattern of inheritance. 
showing a blood pressure of $155 / 87 \mathrm{mmHg}$, heart rate of 114 beats per minute and a respiratory rate of 20 per min. Her body mass index was 21.1. She was found to have atrophic cheeks, prognathism, and facial hirsutism. She also had a prominent umbilicus and a muscular abdomen. There was no hepatomegaly or features of acromegaloid facies.

Her routine laboratory investigations, including complete blood count and serum electrolytes, were within normal limits. Her renal profile showed a creatinine level of $111 \mathrm{mmol} / \mathrm{L}$; otherwise, it was insignificant. Her serum lipid profile was investigated. A lipemic sample was extracted (Figure 3). She was found to have elevated serum triglycerides level of $78.2 \mathrm{mmol} / \mathrm{L}$, and total serum cholesterol was $26.8 \mathrm{mmol} / \mathrm{L}$, low-density lipoprotein cholesterol was $4.2 \mathrm{mmol} / \mathrm{L}$, high-density lipoprotein (HDL) cholesterol was $1.9 \mathrm{mmol} / \mathrm{L}$. Lipopheresis was considered but could not be performed due to unavailability. Her HbA1c was $13.1 \%$. Radiologic findings in the chest were unremarkable.

A genetics specialist saw the patient in our hospital. Genetic testing for the AGPAT2 gene showed a homozygous novel mutation (c.335delC;p.Pro112Argfs*39), which predicted to cause defects in protein function.

We concluded with all the clinical features, investigations, and genetic workup, that the patient was a case of BSCL type 2. She was started on high dose rosuvastatin and gemfibrozil. The patient was carefully followed-up in the outpatient clinic, and the patient's lipid profile improved over time (Figure 4).

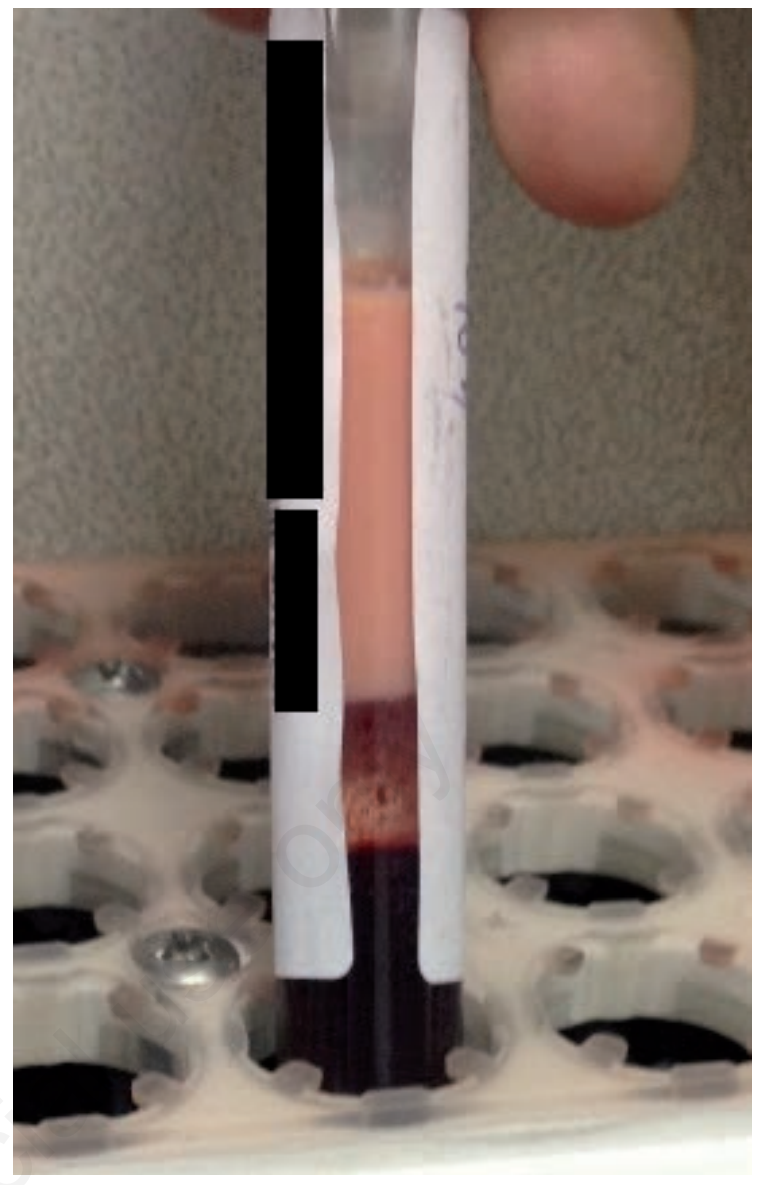

Figure 3. The lipemic sample obtained from the patient.

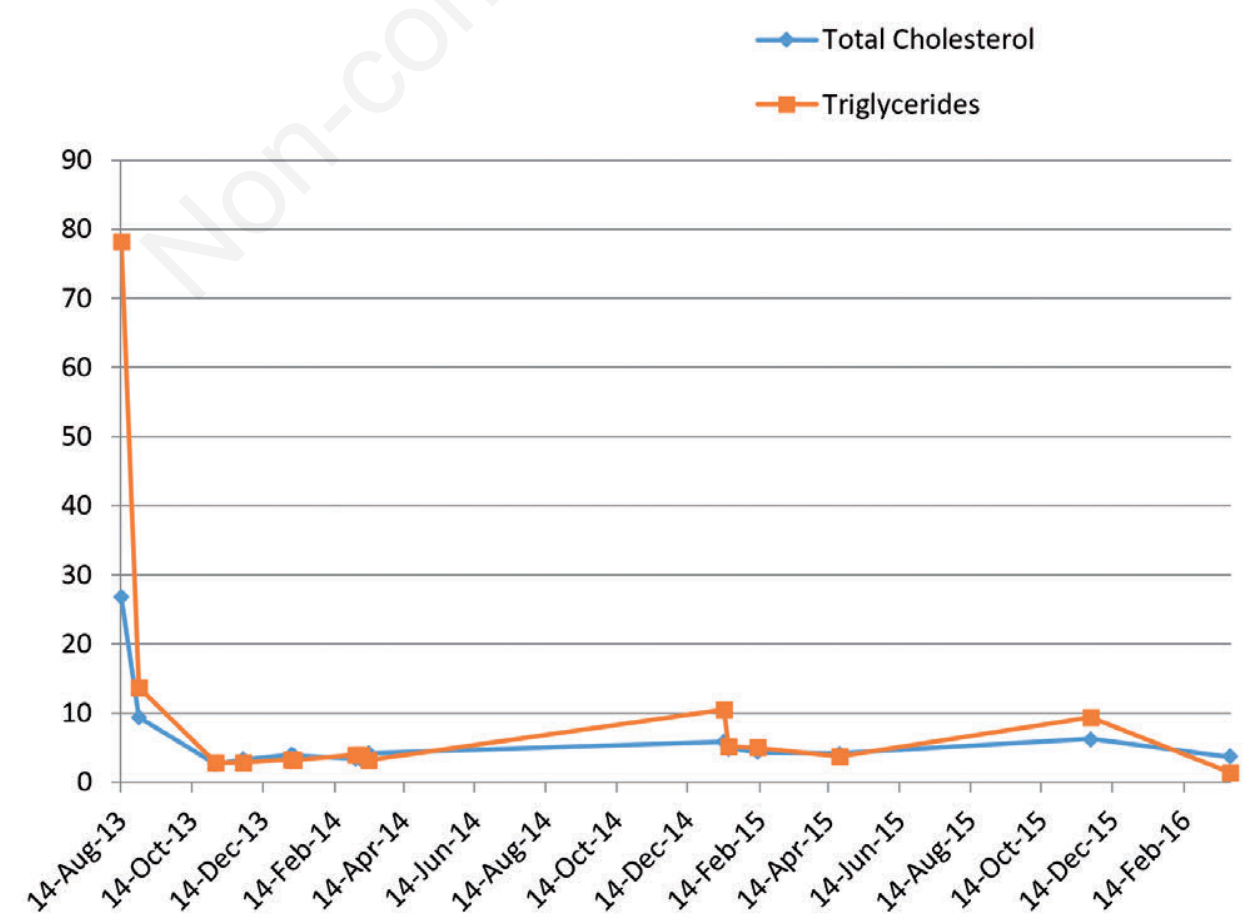

Figure 4. Patient's triglycerides and total cholesterol levels over more than two years of follow-up. 


\section{Discussion}

Clinical presentation, investigations, and genetic testing of our patient are consistent with BSCL type 1. Lipodystrophies are a group of disorders that can be classified according to their origin of being congenital or acquired with a distinctive pattern of generalized or partial. ${ }^{3}$ CGL is characterized by alteration in fat distribution and insulin resistance. Patients present with a lack of body fat at birth or early infancy. Laboratory results of CGL patients show hypertriglyceridemia and low levels of HDL cholesterol. Severe insulin resistance leads to diabetes mellitus in these patients. Absence of adipose tissue results in a hyper muscular appearance. ${ }^{4}$ Leptin deficiency and a defect in adiponectin are being considered as the primary culprits for the metabolic abnormalities associated with BSCL. ${ }^{5}$ Clinical picture may vary from prognathism to acromegaloid features with well-defined musculature and organomegaly in infancy. ${ }^{6}$ Hepatomegaly, hepatic steatosis, and cardiac disorders have been reported frequently in other reports, whereas they were absent in our case. ${ }^{6,7}$ Considering the cutaneous manifestations of the disease, there is a marked variation, including acanthosis nigricans, eruptive xanthomas, and hirsutism, which depends on the severity of the metabolic disorders as well. In our case, these were prominent with the history of xanthomas and the presence of hirsutism. Liver disease and diabetes mellitus are other constant features. Hyperlipidemia usually precedes diabetes. Cardiac, renal and hepatic failure are being attributed as major causes of death among BSCL patients. ${ }^{8}$

Regarding the family pedigree of our patient, the disease manifested in one of the paternal uncles and in one out of four brothers. It is evident that consanguineous marriage is a leading cause of BSCL mutation, as in our case and as reported in multiple cases. ${ }^{9-11}$ Individuals with mutations in AGPAT2 classically originate from sub-Saharan Africa, Northwest Africa, and sporadically from northern Europe and the Middle East. ${ }^{1}$ BSCL type 2 is believed to be more frequent in patients of European and Middle Eastern origins. ${ }^{12}$ The genetic testing of our patient revealed a homozygous mutation in the AGPAT2 gene (c.335delC;p.Pro112Argfs*39), which is predicted to result in frameshift and premature protein termination. To our knowledge, this variant has not been reported in the literature. It is, however, a type of mutation expected to be pathogenic. Therefore, the c.335delC variant is likely the cause of disease in our case.

Treating patients with a strict diet is primitive in the management of BSCL. For control of dyslipidemia, restriction of total fat intake and a calorie-controlled regimen is crucial. Severe hypertriglyceridemia, like in our case, may be controlled by statins and fenofibrates. ${ }^{13}$ Metformin can be considered as an option for treatment of BSCL as it controls the glycidic metabolism as well as contributing for an appetite reduction and improvement of hepatic steatosis and polycystic ovary syndrome. In some cases, like in our patient, insulin therapy may also be necessary to improve the management of type 2 diabetes. ${ }^{3}$ Studies with leptin replacement therapy on controlling hyperglycemia and hypertriglyceridemia associated with BSCL have been done. One of those studies shows that metreleptin treatment is a potential treatment for these metabolic disorders associated with lipodystrophy. ${ }^{14}$

\section{Conclusions}

We report a very rare case of an adult patient with BSCL type 1, the first of its kind in Saudi Arabia. The genetic testing showed a novel sequence variant designated as c. $335 \mathrm{delC}$ in the $A G P A T 2$ gene, which we believe was the cause of our patient's disease. Many limitations exist as BSCL is an infrequent condition and limited literature is available. To overcome BSCL treatment challenges, more patients should be studied and more reports need to be published.

\section{References}

1. Van Maldergem L, Magre J, Khallouf TE, et al. Genotype-phenotype relationships in Berardinelli-Seip congenital lipodystrophy. J Med Genet 2002;39:722-33.

2. Babu P, Sharma R, Jayaseelan E, Appachu D. Berardinelli-Seip syndrome in a 6-year-old boy. Indian J Dermatol Venereol Leprol 2008;74:644-6.

3. Garg A. Acquired and inherited lipodystrophies. N Eng1 J Med 2004;350:1220-34.

4. Agarwal AK, Simha V, Oral EA, et al. Phenotypic and genetic heterogeneity in congenital generalized lipodystrophy. J Clin Endocrinol Metab 2003;88:4840-7.

5. Haque WA, Shimomura I, Matsuzawa Y, Garg A. Serum adiponectin and leptin levels in patients with lipodystrophies. J Clin Endocrinol Metab 2002;87:2395.

6. Garg A. Clinical review: lipodystrophies: genetic and acquired body fat disorders. J Clin Endocrinol Metab 2011;96:3313-25.

7. Pelosini C, Martinelli S, Bagattini B, et al. Description of an AGPAT2 pathologic allelic variant in a 54-yearold Caucasian woman with Berardinelli-Seip syndrome. Acta Diabetol 2011;48:243-6.

8. Garg A. Lipodystrophies. Am J Med 2000;108:143-52.

9. Faria CA, Moraes RS, Sobral-Filho DC, et al. Autonomic modulation in patients with congenital generalized lipodystrophy (Berardinelli-Seip syndrome). Europace 2009;11:763-9.

10. Metwalley KA, Farghaly HS. Berardinelli-Seip syndrome type 1 in an Egyptian child. Indian J Hum Genet 2014;20:75-8.

11. Prasad CA. Berardinelli Seip syndrome. Med J Armed Forces India 2006;62:83-4. 
12. Magre J, Delepine M, Khallouf E, et al. Identification of the gene altered in Berardinelli-Seip congenital lipodystrophy on chromosome 11q13. Nature Genet 2001;28:365-70.

13. Gomes KB, Pardini VC, Fernandes AP. Clinical and mo- lecular aspects of Berardinelli-Seip congenital lipodystrophy (BSCL). Clinica Chimica Acta 2009;402:1-6.

14. Chan JL, Lutz K, Cochran E, et al. Clinical effects of long-term metreleptin treatment in patients with lipodystrophy. Endocr Pract 2011;17:922-32. 